\title{
Changes in Cardiac Autonomic Regulation after Acute Lung Exposure to Carbon Nanotubes: Implications for Occupational Exposure
}

\author{
Jacopo M. Legramante, ${ }^{1,2,3,4}$ Sergio Sacco, ${ }^{1,3,4}$ Patrizio Crobeddu, ${ }^{1,2,3,4}$ Andrea Magrini, ${ }^{5}$ \\ Federica Valentini, ${ }^{6}$ Giuseppe Palleschi, ${ }^{6}$ Marco Pallante, ${ }^{1,4}$ Rita Balocchi, ${ }^{7}$ Ivo Iavicoli, ${ }^{8}$ \\ Antonio Bergamaschi, ${ }^{8}$ Alberto Galante, ${ }^{1,2,3}$ Luisa Campagnolo, ${ }^{9}$ and Antonio Pietroiusti ${ }^{5}$
}

\author{
${ }^{1}$ Dipartimento di Medicina Interna, Università “Tor Vergata”, Via Montpellier 1, 00133 Roma, Italy \\ ${ }^{2}$ A.F.O. Medicina d'Urgenza-Pronto Soccorso, Dipartimento delle Emergenze, Policlinico Tor Vergata, Viale Oxford 81, \\ 00133 Roma, Italy \\ ${ }^{3}$ Divisione di Riabilitazione Cardiologica, IRCCS S. Raffaele, Via dei Laghi Km 19.600, 00049 Velletri, Italy \\ ${ }^{4}$ Stazione per la Tecnologia Animale, Università “Tor Vergata”, Via Montpellier 1, 00133 Roma, Italy \\ ${ }^{5}$ Sezione di Medicina del Lavoro, Dipartimento di Biopatologia e Diagnostica per Immagini, Università di Roma "Tor Vergata", \\ Via Montpellier 1, 00133 Rome, Italy \\ ${ }^{6}$ Dipartimento di Scienze e Tecnologie Chimiche, Università di Roma "Tor Vergata”, Via della Ricerca Scientifica 1, \\ 00133 Roma, Italy \\ ${ }^{7}$ CNR-Centro Nazionale delle Ricerche, Istituto di Fisiologia Clinica, Via Giuseppe Moruzzi 1, 56124 Pisa, Italy \\ ${ }^{8}$ Istituto di Medicina del Lavoro, Università Cattolica del Sacro Cuore, Largo F. Vito 1, 00168 Roma, Italy \\ ${ }^{9}$ Dipartimento di Salute Pubblica e Biologia Cellulare, Università “Tor Vergata”, Via Montpellier 1, 00133 Roma, Italy
}

Correspondence should be addressed to Antonio Pietroiusti, pietroiusti@med.uniroma2.it

Received 14 October 2011; Accepted 20 February 2012

Academic Editor: Sergio Iavicoli

Copyright () 2012 Jacopo M. Legramante et al. This is an open access article distributed under the Creative Commons Attribution License, which permits unrestricted use, distribution, and reproduction in any medium, provided the original work is properly cited.

Carbon nanotubes (CNTs) are among the most relevant engineered nanomaterials (ENMs). Given the expected rise of exposure to ENMs, there is concern that they may adversely affect health of exposed people. Aim of the study was to test the hypothesis that single wall carbon nanotubes (SWCNTs) pulmonary exposure acutely affect the autonomic cardiovascular regulation in conscious rats. We studied Wistar-Kyoto rats in which a telemetry transmitter for continuous arterial pressure (AP) and heart rate (HR) recordings was surgically implanted. SWCNTs dispersed in phosphate buffer saline (PBS) or PBS alone were randomly administered intratracheally. Immediately before, and 24 hours after each instillation a $30 \mathrm{~min}$ AP recording was performed. The sequence analysis was performed to evaluate the baroreflex function. In the control group, PBS instillation did not induce any significant changes. At variance the SWCNT exposure induced a significant reduction of baroreflex system (BRS) $(3.5 \pm 0.6$ versus $2.6 \pm$ $0.40 \mathrm{msec} / \mathrm{mmHg}$ ) without significant changes in the occurrence of baroreflex sequences $(7.5 \pm 0.47 \%$ versus $7.4 \pm 0.38 \%)$. Our results show that SWCNT pulmonary exposure might affect the cardiovascular autonomic regulation thus contributing to cardiac and arrhythmic events.

\section{Introduction}

There is strong evidence that episodic high levels of airborne particulate matter (PM) are associated with stroke, heart attacks, heart arrhythmias, and sudden death [1]; these events may be precipitated, at least in part, by alterations in the autonomic input to the heart [2-5], which can be displayed by a partial or total loss in spontaneous heart rate variability (HRV). Indeed, decreased HRV is highly predictive for increased risk of arrythmias and sudden cardiac death [6], especially in subjects with ischemic heart disease [7]. 
There are also reports showing that higher levels of ambient air pollutants increase the risk of emergency department (ED) visits for cardiac arrhythmia [8]. Moreover it has been suggested that alterations in the autonomic tone eventually due to increased environment air pollution might contribute to the instability of a vascular plaque or initiate cardiac arrhythmias thus representing a plausible explanation for the occurrence of acute cardiovascular events, such as increased myocardial infarctions [9].

However, previous studies on PM have shown that ultrafine (UF) particles are more cytotoxic, inflammatory, and fibrogenic on an equivalent mass basis than fine-sized particles of the same composition. It is therefore not surprising that a recent large European study showed that the ultrafine (UF) component of PM is the major factor contributing to the alterations of cardiovascular autonomic control [10]. The responsible underlying mechanisms remain unclear but may involve activation of pulmonary neural reflex arcs, direct effects of pollutants on cerebral areas responsible for autonomic control or on cardiac ion channels, or may follow the heightened systemic inflammatory state.

Synthetic nanomaterials, developed in recent years to engineer new structures, materials, and devices, generally occur in size ranges similar to UF particles. An important class of synthetic nanomaterials is represented by carbon nanotubes. These cylindrical carbon molecules have novel properties, making them potentially useful in many applications in nanotechnology, electronics, optics, and other fields of materials science, as well as potential uses in architectural fields. Therefore, their wide utilization raises concern about possible adverse effects on cardiovascular system. Indeed, some preliminary animal data suggest that carbon nanotubes may promote atherosclerosis [11].

No definitive data are currently available on their possible effects on the cardiovascular autonomic control.

The introduction of techniques able to monitor arterial pressure and heart rate in conscious, freely moving animals allows to evaluate possible modifications of autonomic cardiovascular regulation over time in response to various experimental conditions [12]. In particular, the possibility to monitor the baroreflex control of sinus node by studying the baroreflex sensitivity (BRS), in a noninvasive and nonobtrusive way [13], is really intriguing due to its prognostic value in cardiovascular diseases $[14,15]$.

Therefore aim of the present study was to test the hypothesis that single wall carbon nanotubes (SWCNTs) pulmonary exposure in a physiological model of conscious rat acutely affect the autonomic cardiovascular regulation and in particular the arterial baroreflex function which has been widely considered as a predictive index of cardiac mortality in patients affected by ischemic heart disease and myocardial infarction $[14,15]$.

\section{Material and Methods}

2.1. SWCNT Preparation. SWCNTs (CarboLex, Aldrich, Steinheim, Germany) produced by the arc-discharge process, employing $\mathrm{CO}$ in a continuous-flow gas phase as the carbon

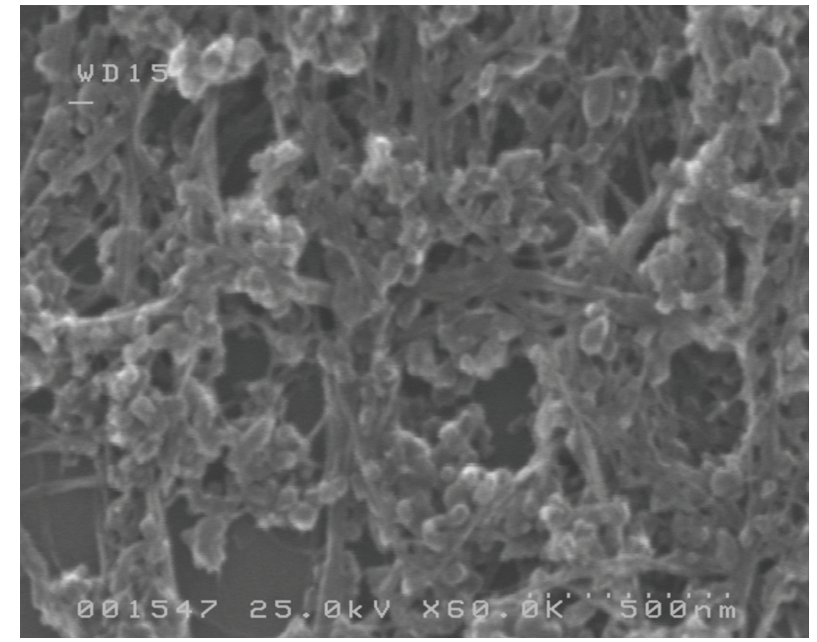

FIGURE 1: Scanning electron microscopy imaging of SWCNTs administered to rats. A high degree of agglomeration is evidenced.

feedstock and $\mathrm{Fe}(\mathrm{CO})_{5}$ as the iron-containing catalyst precursor and purified by the $\mathrm{HNO}_{3}$ acid treatment at room temperature, were used in this study. For the SWCNTs morphological and structural characterization, Raman spectroscopy and scanning electron microscopy (SEM) were routinely used to characterize the as-received nanotube samples and the samples submitted to the purification processing steps. The Raman spectroscopy revealed a typical spectrum associated with the radial breathing mode (RBM) of carbon atoms, representing the fingerprint of single-walled carbon nanotubes. The analysis of the Raman signals in the tangential modes of the graphite lattice allowed information about the phase purity of the purified carbon nanotubes. This last showed a Raman spectrum unaffected by the acidic treatments.

\subsection{Physicochemical Characterization. Purified suspended} SWCNTs were used in the study at the final concentration of $1 \mathrm{mg} / \mathrm{mL}$. The range of diameter and length and surface area of SWCNTs were $1.2-1.6 \mathrm{~nm}, 2-5 \mu \mathrm{m}$, and $300 \mathrm{~m}^{2} / \mathrm{g}$, respectively. Surface area was determined by Brunauer, Emmett, and Teller analysis (BET method). Carbon nanotubes were suspended in phosphate-buffered saline (0,1 M PBS, pH 7.4); immediately before the pulmonary exposure, they were sonicated for 10 minutes with a Branson Sonifier B-12 (Cell Disruptor, Sonic Power Company Danbury Connecticut at $100 \mathrm{~W}$ ) in order to obtain a better dispersion of the nanomaterial. The choice to disperse SWCNTs in PBS was taken in order to reproduce the same experimental conditions of a previous relevant study showing a proatherosclerotic effect after intratracheal instillation of SWCNTs [11]; the $10 \mathrm{~min}$ sonication procedure was chosen because in preliminary experiments we found that after $10 \mathrm{~min}$ sonication, the dispersion remained stable up to $1 \mathrm{~min}$. However, as shown in Figure 1, a high degree of agglomeration was detectable at scanning electron microscopy. During all the instillation experiments, the purified SWCNT dispersion was subjected 
Baroreflex sequences
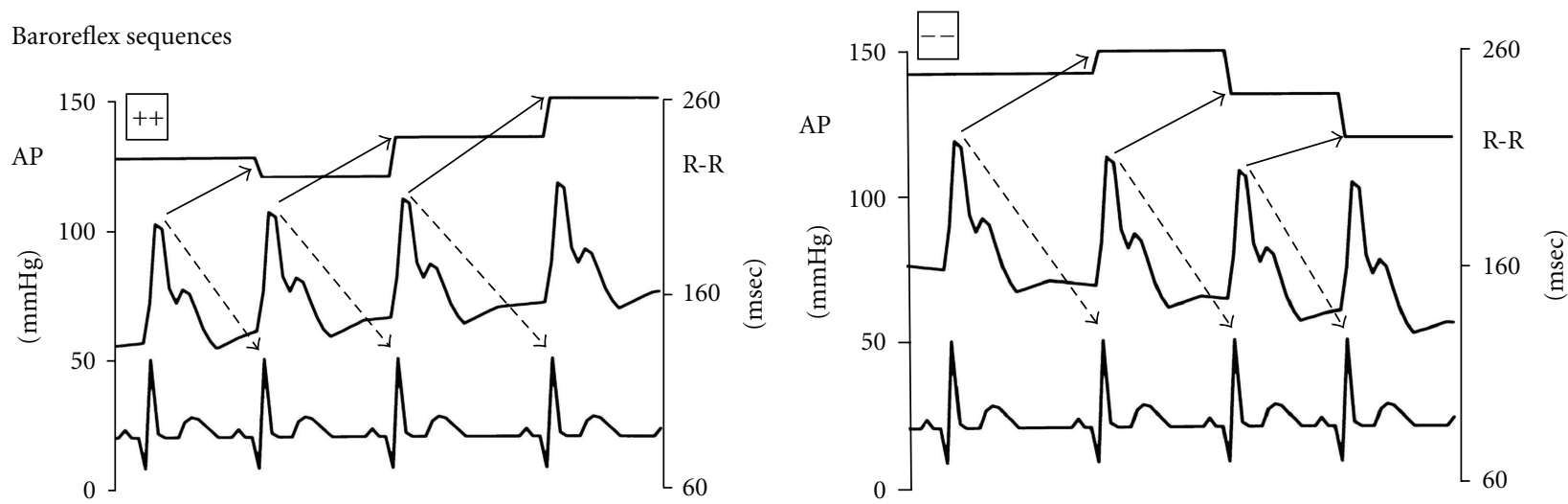

FIgURE 2: Examples of the arterial pressure, ECG signals and of the corresponding pulse intervals. The signals refer to sequences during which SAP and PI of the fifth heart beat (i.e., lag 5, used for the high heart rate of rats, see Section 2 for details) changed in the same direction, either increasing, hypertension/bradycardia, or decreasing, hypotension/tachycardia (baroreflex sequences).

to the ultrasonic bath (LBS2, $135 \mathrm{~W})$ for 10 minutes, and instilled intratracheally within 1 minute after sonication, to ensure stability of the homogeneous SWCNT dispersion. The amount of SWCNTs given during each session was $1 \mu \mathrm{g} / \mathrm{gr}$ body weight. This concentration is in the lower range of the dosage given in previous experimental studies aimed at assessing lung and cardiovascular toxicology of SWCNTs [11, $16,17]$.

2.2.1. General Procedures. The study was performed on 16 adult Wistar-Kyoto (WKY) rats of both sexes ( $350 \mathrm{~g}$ body wt) and the experimental procedures were carried out according to the Association for Assessment and Accreditation of Laboratory Care International and approved by the animal care facility (Stazione per la Tecnologia Animale) of the University "Tor Vergata" and by the Italian Health Minister. Rats were used and housed individually in the animal care facility, allowed to normal rat chow and drinking water ad libitum, and kept on a $12 \mathrm{~h}$ light- $12 \mathrm{~h}$ dark cycle.

2.2.2. Surgical Procedures. As previously described [13], after having induced the anesthesia by Ketamine (Ketavet 50 $60 \mathrm{mg} / \mathrm{kg}$ i.p.) and Medetomidine (Domitor $0.3 \mathrm{mg} / \mathrm{kg}$ i.p.) a telemetry transmitter (TA11PA-C40, Data Sciences, St. Paul, MN, USA) was implanted for recordings of AP signals according to manufacturer specifications. The tip of the arterial catheter was inserted into the abdominal aorta previously exposed by a midline incision via a hole made by a 21 -gauge needle below the bifurcation of the renal arteries just proximal to the iliac bifurcation and secured in place with tissue glue (Vetbond, $3 \mathrm{M}$ ). The transmitter body was attached to the abdominal wall along the incision line with sutures as the incision was closed. After surgery, the rats were given antibiotics (ceftriaxone) and housed individually in cages for 5-7 days of recovery before any experimental protocol began.

\subsubsection{Measurement of Arterial Pressure Using Radiotelemetry.} As previously reported [13] the system used to record arterial pressure consists of three basic elements: (1) a transmitter for monitoring BP (TA11PAC40); (2) a receiver (RPC-1); (3) an adapter (R11CPA) with an ambient pressure monitor (APR1) that produces analog output signals of pulsatile AP. The telemetered AP signal was digitized using an analog I/O PC card (National Instrument 6024E, Austin, TX, USA) at a rate of $2000 \mathrm{~Hz}$, displayed on the computer screen and processed by an algorithm based on feature extraction to detect and measure the characteristics of AP cycles developed in our laboratory based on a Lab view platform software. Pulse interval (PI) was measured from the pressure pulses and used to calculate heart rate (HR).

2.2.4. Experimental Protocol. Rats were randomly divided into two groups: control rats $(n=7)$ and SWCNT instilled rats $(n=9)$. SWCNTs or PBS were given to SWCNT instilled and control group, respectively. Briefly, after having induced the anesthesia as previously described, the trachea was intubated with a polyethylene cannula of the same diameter and the instillation was performed. Rats in both groups underwent 30 min duration AP recordings performed in baseline conditions and 24 hours after the instillation.

2.3. Baroreflex Analysis by "Sequence Technique". The sequence analysis was performed as previously reported $[13,18]$. Briefly, the beat-by-beat time series of systolic arterial pressure (SAP) and PI were analyzed by a computer to identify spontaneously occurring sequences of three or more consecutive beats in which SAP and PI of the fifth heart beat (i.e., lag 5, used for the high heart rate of rats) [19] changed in the same direction, for example, SAP increasing and PI increasing (i.e., hypertension and bradycardia) or SAP decreasing and PI decreasing (i.e., hypotension and tachycardia). These sequences were identified as "baroreflex" sequences (Figure 2). A linear regression was applied to each individual sequence, similarly to the Oxford technique employing bolus injections of vasoactive drugs. Only those sequences in which $r^{2}$ was $>0.85$ were accepted and the number of baroreflex sequences was calculated. 
The mean individual slope of the baroreflex sequences, obtained by averaging all slopes computed within a given experimental period, was calculated and taken as a measure of the baroreflex sensitivity (BRS) for that period [18].

The engagement time was also calculated as the fractional occurrence of the sequences independently on recording time and/or HR as previously reported [13]. Briefly, this index has been obtained by dividing the sum of the R-R organized in sequences, according to the criteria reported above, by the total recording duration and multiplying it by 100.

2.4. Power Spectral Analysis. The heart rate variability (HRV) was assessed in the frequency domain by computing the power spectral density (PSD) in selected frequency bands.

As expected, dealing with conscious and freely moving animals, the 30 min RR recordings showed periods of nonstationarities both in mean and standard deviations. To comply with the requirements of the analysis we first processed all the recordings to extract stationary epochs (in weak sense). To this aim a mixed, automatic and visual, procedure was applied. First, mean, standard deviation (SD) and slope of the linear trend were computed over nonoverlapping intervals of $1 \mathrm{~min}$ spanning the whole recording. The selection of stationary intervals was then performed by visual inspection of the time course of mean, SD, and slope values. Stationary intervals of different lengths were detected inside the recordings: for uniformity of analysis a $5 \mathrm{~min}$ stationary interval was selected for each animal.

Spectral estimation was performed using Welch's averaged modified periodogram method with a Hamming window and a minimum of $50 \%$ epochs overlap. The variable overlap comes from the choice of having, for each animal, at least a preset minimum number of epochs to average. PSD was assessed in the frequency range of $0.2-6 \mathrm{~Hz}$ involving very low frequency (VLF: from 0 to $0.2 \mathrm{~Hz}$ ), low frequency (LF: from 0.2 to $0.6 \mathrm{~Hz}$ ), and high frequency (HF: from 0.6 to $3.0 \mathrm{~Hz}$ ) spectral bands [20]. According to the literature, the PSD values VLFn, LFn, and HFn, expressed in normalized units, are obtained as the ratio between the VLF, LF, and HF powers and the cumulative power LF $+\mathrm{HF}$, respectively, $[21,22]$.

In addition, the ratio of low-frequency (LF) to high-frequency modulation (HF) of the RR intervals which is considered to be an indirect marker of the sympathovagal balance (LF/HF) was also assessed [7]. All the software code was written in Matlab 7.10.

2.5. Data Analysis and Statistics. AP data were stored and analyzed by a computerized on line system for biological data elaboration developed in our laboratory based on a Lab view platform software.

Within groups changes in the reported variables were evaluated by one-way ANOVA for repeated measures for normally distributed variables and by Kruskal-Wallis one way ANOVA on Ranks for nonnormally distributed variables. The significance of differences of baseline values between the two experimental groups was evaluated by $t$-test. All data are
TABLE 1: Comparison of cardiovascular and autonomic values in baseline (i.e., before intratracheal instillation of either PBS or SWCNTs) condition between control and SWCNT instilled rats.

\begin{tabular}{lccc}
\hline & Control $(n=7)$ & \multicolumn{2}{c}{ Instilled $(n=9)$} \\
\hline SAP (mmHg) & $130.7 \pm 5.1$ & $127.2 \pm 5.0$ & NS \\
DAP (mmHg) & $99.9 \pm 4.4$ & $95.8 \pm 3.2$ & NS \\
HR (b/min) & $333.1 \pm 17.0$ & $351.6 \pm 12.0$ & NS \\
BARO $\%$ & $8.3 \pm 0.7$ & $7.5 \pm 0.5$ & NS \\
BRS (msec/mmHg) & $4.1 \pm 0.9$ & $3.5 \pm 0.6$ & NS \\
NBARO\% & $4.1 \pm 0.5$ & $3.9 \pm 0.2$ & NS \\
NBRS $(\mathrm{msec} / \mathrm{mmHg})$ & $4.1 \pm 0.9$ & $3.4 \pm 0.5$ & NS \\
\hline
\end{tabular}

Mean values \pm SEM. SAP: systolic arterial pressure; DAP: diastolic arterial pressure; HR: heart rate; BARO\%: engagement time for baroreflex sequences; BRS: baroreflex sensitivity; NBARO\%: engagement time for nonbaroreflex sequences; NBRS: gain of the nonbaroreflex sequences. Statiscal analysis has been performed by one-way repeated measures analysis of variance or Friedman repeated measures analysis of variance on ranks tests.

presented as means \pm SEM. A value of $P<0.05$ was considered statistically significant.

\section{Results}

In baseline conditions (i.e., before intratracheal instillation of either PBS or SWCNTs) cardiovascular parameters did not differ between control and SWCNT instilled rats (Table 1) and no significant cardiovascular changes were evident after 24 hours (Figure 3 ) in both experimental groups.

Similarly the occurrence of baroreflex sequences and BRS in baseline conditions did not show significant changes between control and SWCNT instilled rats (Table 1). Also the indexes evaluating the spectral analysis of the heart rate variability were similar in baseline condition between the two experimental groups and in particular LFn was $0,079 \pm 0,01$ for control group and 0,066 $\pm 0,009$ for SWCNT instilled rats, HFn 0,402 $\pm 0,06$ versus $0,261 \pm 0,05$, VLFn $0,519 \pm$ 0,07 versus $0,673 \pm 0,05$, and $L F n / H F n ~ 0,209 \pm 0,02$ versus $0,342 \pm 0,07$.

The power spectral analysis of the heart rate variability did not appear to be affected by the SWCNT experimental acute exposure. In fact LFn, HFn, VLFn, LFn/HFn did not show significant changes between basal condition and after 24 hour from the instillation both in the control and in the SWCNT instilled rats (Figure 4).

In the control group the PBS instillation did not induce any significant changes in the occurrence and in the sensitivity of baroreflex sequences (Figure 5). At variance the SWCNT experimental acute exposure induced a significant reduction of BRS without significant changes in the occurrence of baroreflex sequences (Figure 5). These differences are not trivial because whereas in SWCNT instilled rats the significant reduction accounts about $21 \%$ for BRS, in the control group BRS shows an increase of about $51 \%$. Indeed the BRS percent changes from baseline versus acute exposure are significantly different between PBS and SWCNT instilled rats (Figure 6). 

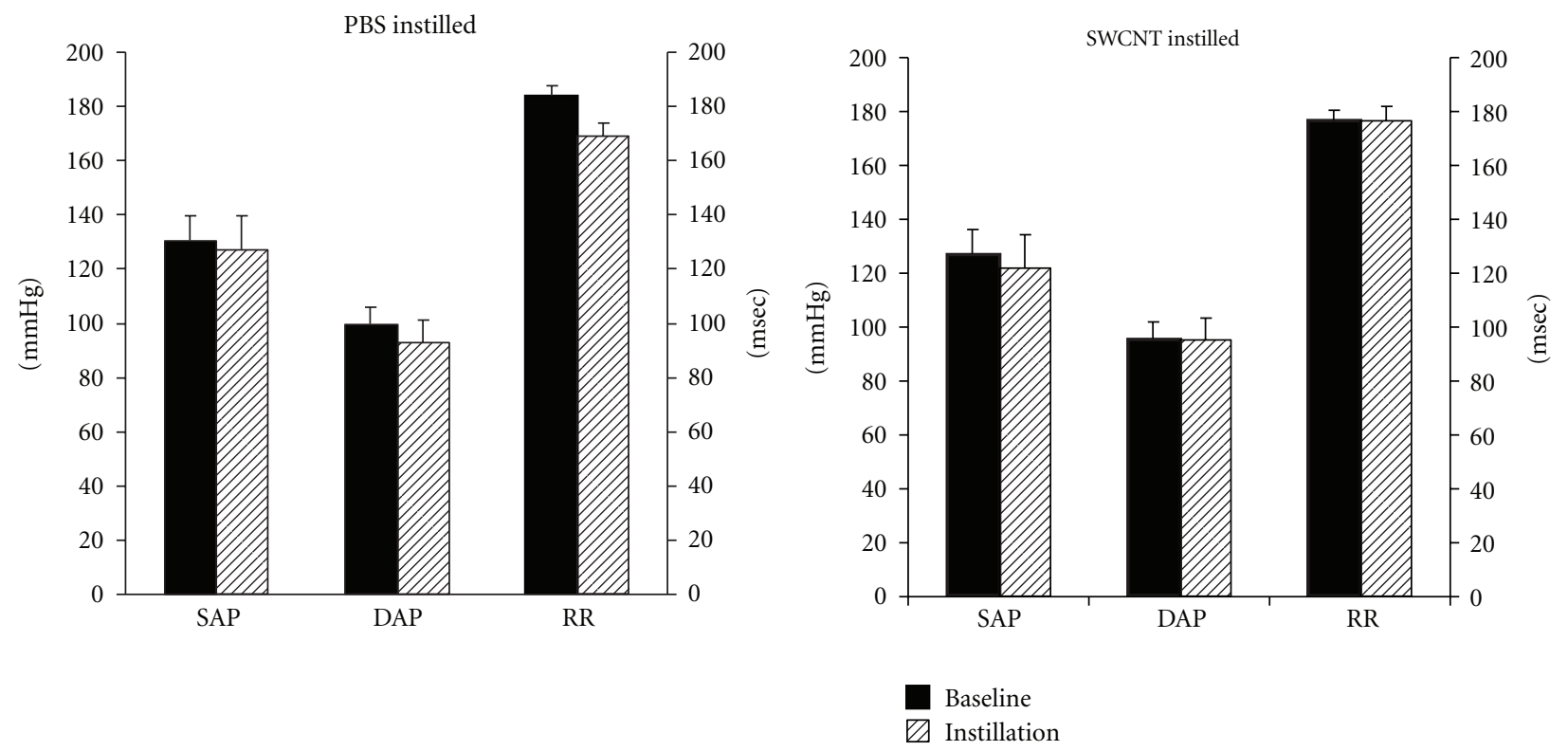

FIGURE 3: Systolic (SAP), diastolic (DAP) arterial pressure, and RR interval values before and after acute instillation of, respectively, PBS (control group) and SWCNTs (experimental group).
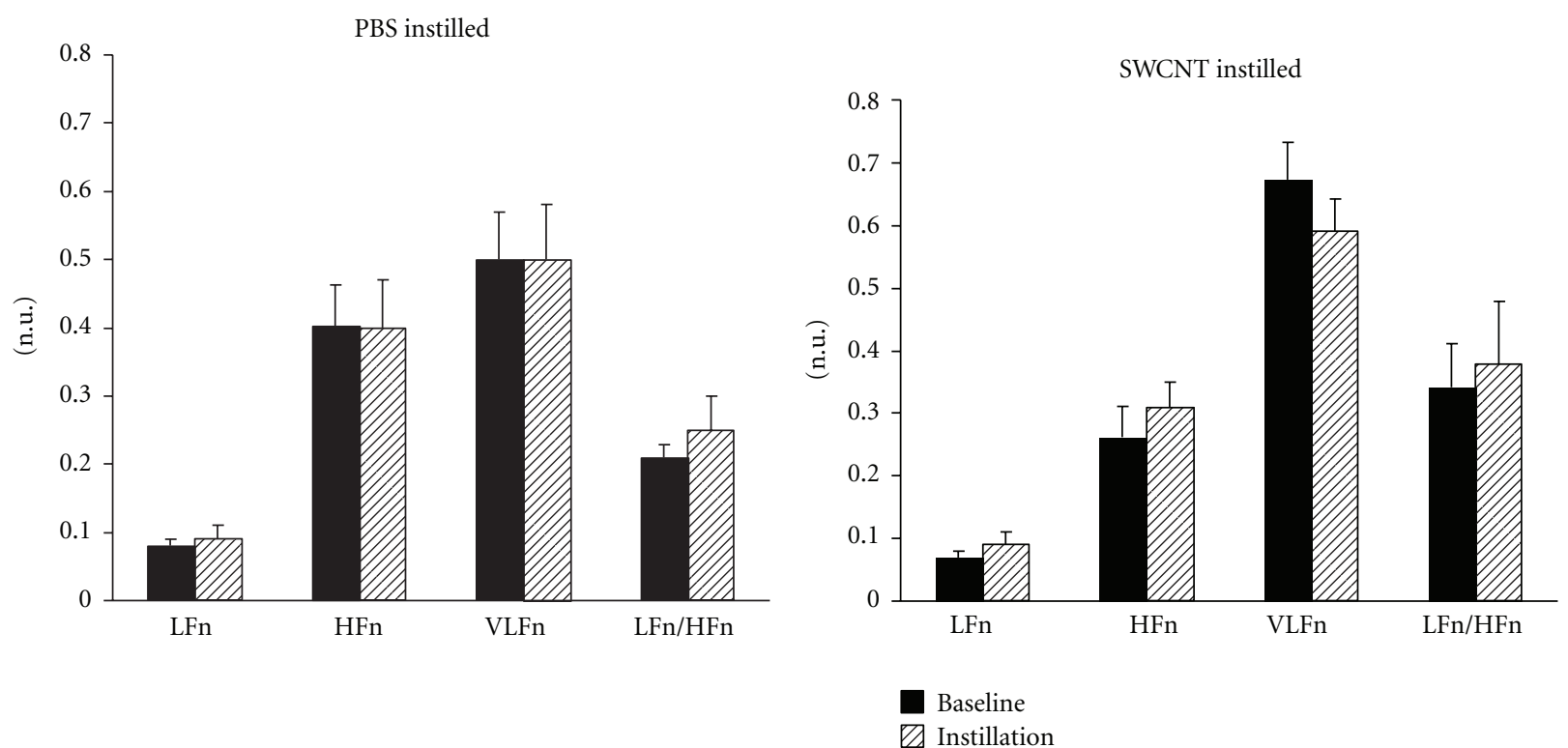

Figure 4: Differences in the frequency domain indexes of heart rate variability obtained by computing the power spectral density (see Section 2 for the details) in response to acute instillation of, respectively, PBS (control group) and SWCNTs (experimental group). LFn: low frequency values expressed in normalized units; HFn: high frequency values expressed in normalized units; VLFn: very low frequency values expressed in normalized units; LFn/HFn: the ratio of normalized low-frequency (LF) to high-frequency modulation (HF) of the RR intervals which is considered to be an indirect marker of the sympathovagal balance (LFn/HFn).

The mean prevailing systolic arterial pressure determining the operating point at which the baroreflex and nonbaroreflex sequences were calculated did not differ significantly after the instillation both in the control and in the experimental group $(127,2 \mathrm{mmHg}$ in baseline condition versus 121,9 mmHg after SWCNT instillation and 130,7 mmHg in baseline condition versus $127,4 \mathrm{mmHg}$ after the instillation in control group).

\section{Discussion}

The main goal of the present investigation was to study the autonomic cardiovascular regulation in response to acute pulmonary exposure to carbon nanotubes. The distinctive aspect of the present study has been to utilize a physiological model of conscious freely moving rats in order to avoid any possible and well known interference of anesthetic drugs 

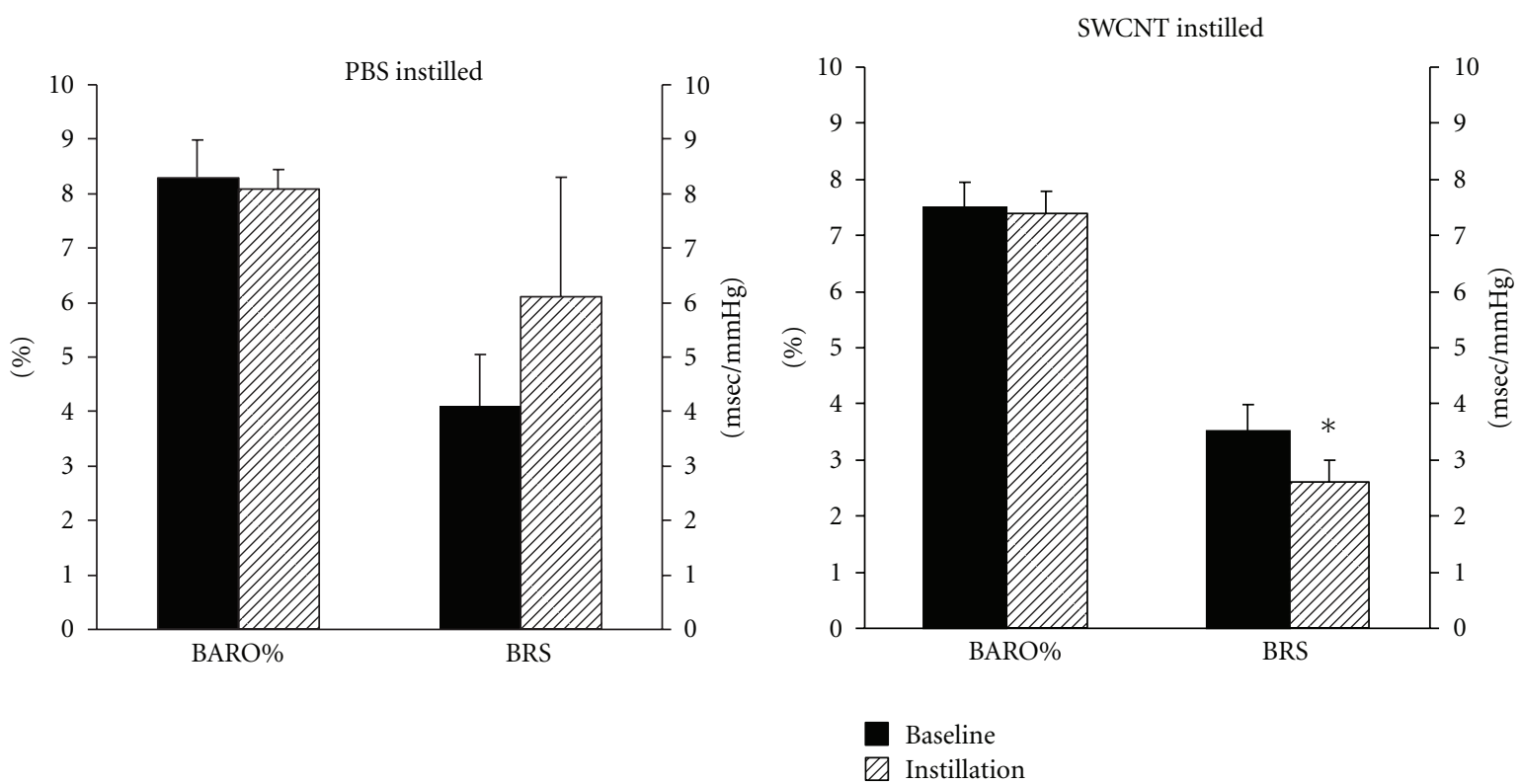

FIGURE 5: Occurrence of baroreflex sequences (BARO\%), baroreflex sensitivity (BRS) before and after acute instillation of, respectively, PBS (control group) and SWCNTs (experimental group). Asterisk indicates a significant difference $(P<0.05)$.

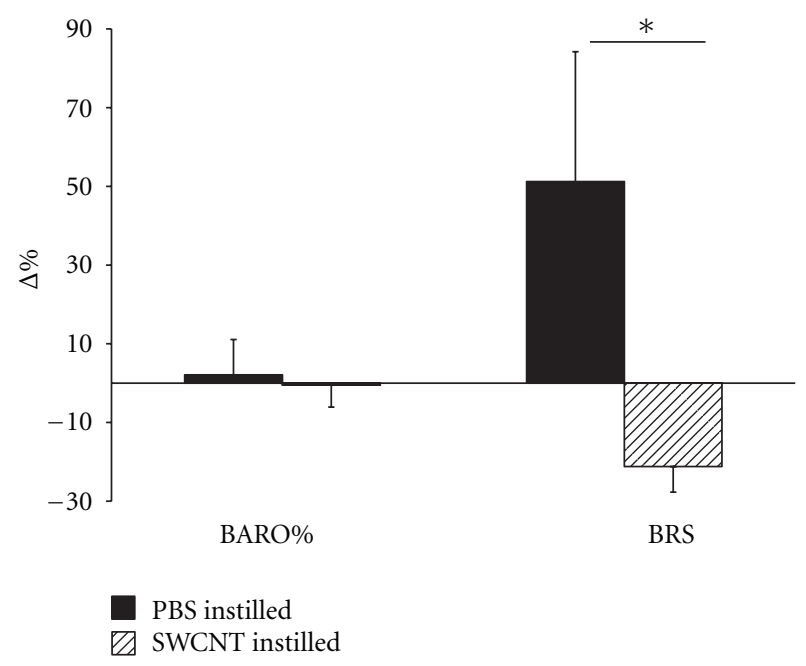

Figure 6: Percent changes of baroreflex sequences (BARO\%), baroreflex sensitivity (BRS) after acute instillation of, respectively, PBS (control group) and SWCNTs (experimental group) from baseline values. Asterisk indicates a significant difference $(P<0.05)$.

and other experimental tools routinarily used in acute experimental models.

It has been largely reported that airborne particulate matter (PM) is associated with cardiovascular diseases and in particular with acute events thus increasing the ED visits [8]. Moreover UF particles seem to cause an alteration of the cardiovascular autonomic regulation [10]. Less data exist concerning the effect of synthetic nanomaterials even though their size is similar to UF particles. In particular the attractive properties of nanomaterials for medical and technological applications induces an increase in their utilizations and therefore a growing level of possible exposure to these materials.

Concerning the cardiovascular system, previous studies have shown that carbon nanotubes have the potential effect of promoting atherosclerosis [11].

Our study shows for the first time that acute pulmonary exposure to SWCNTs causes a reduction of BRS in conscious freely moving rats.

Infact in the control group PBS instillation induced a non significant increase in BRS whereas in the experimental group SWCNT instillation produced a significant BRS reduction. Even when considered as percent change from control values, the responses to PBS and to SWCNTs resulted significantly different despite the relevant data dispersion as demonstrated by SEM (Figure 6).

In the intact circulation beat-by-beat spontaneous arterial pressure fluctuations have been shown to be linked to beat-by-beat spontaneous changes in RR interval through baroreflex mechanisms. On this basis the "sequence" technique [23] is able to extract from SAP and RR time series sequences of spontaneously occurring consecutive beats in which SAP and RR of the following beat change in the same direction, that is hypertensive/bradycardic $(\mathrm{SAP}+/ \mathrm{RR}+)$ and hypotensive/tachycardic sequences (SAP-/RR-). These sequences have been named "baroreflex sequences" and have been considered as expression of negative feedback mechanisms of baroreflex origin [2] (Figure 2).

The introduction of techniques able to monitor arterial pressure and heart rate in conscious freely moving animals allows to evaluate possible modifications of autonomic cardiovascular regulation over time in response to various experimental conditions [12]. In particular, the possibility to monitor the baroreflex control of sinus node by studying the baroreflex sensitivity (BRS), in a noninvasive and 
nonobtrusive way [13] is really intriguing due to its prognostic value in cardiovascular diseases [15]. Indeed a pilot study from our group suggested the feasibility to evaluate the arterial baroreflex function [24] in response to SWCNT pulmonary exposure in conscious freely moving rats. Moreover, autonomic cardiovascular regulation has been investigated by means of power spectral analysis of HR and BP variability, a technique currently used to derive noninvasive indexes of the different neural components modulating the sinoatrial node and the autonomic vascular outflow [7].

The possibility to analyze the cardiovascular autonomic pattern and in particular the arterial baroreflex function is relevant because it has been widely shown that alteration in the autonomic modulation of the cardiovascular system has an important prognostic and predictive value in patients affected by cardiovascular disease. In particular several studies have indicated that a depressed BRS, an index of the reflex vagal control of the sinus node is associated with a greater risk for ventricular fibrillation during transient ischemia after myocardial infarction $[25,26]$. The unfavorable effect of a depressed BRS on ventricular arrhythmias during myocardial ischemia also extends to animals without prior myocardial infarction [26]. Moreover, clinical studies have clearly demonstrated that BRS is markedly depressed during spontaneous episodes of transient myocardial ischemia [27] and in patients without a myocardial infarction who experienced out-of-hospital ventricular fibrillation [28].

Therefore the possibility that the reduced BRS in response to SWCNT acute exposure, shown in the present study, might contribute to the increased cardiovascular risk connected to high levels of airborne particulate matter, as constituted both by PM and UF matter, must be taken into account. Indeed increased levels of environmental UF particulate have been reported to be linked to increased ED access for cardiac arrhythmias [8].

It is noteworthy that the power spectral analysis has not shown significant changes in response to acute SWCNT exposure. This is an important information, because BRS and power spectral analysis, as tools to extract physiological information from the heart rate variability, reflect different, although possibly related [29], aspects of the autonomic modulation of the heart rate and are not clinically redundant $[15,30]$. Although both BRS and heart rate variability effectively discriminate individuals at high and low risk for cardiac mortality [15], experimental [31], and clinical [32-34] studies have indicated that BRS is more useful in the prediction of future cardiac events in patients affected by ischemic cardiac disease. Another possible alternative explanation is that our analysis has been performed on 5-min intervals, a time in which is possible to evaluate only the short-term HRV thus loosing information that could be present in the whole-time interval. Furthermore, it must be pointed out that power spectral analysis is a tool belonging to the field of the so-called linear analysis and does not take into account possible nonlinear variations occurring between the two conditions.

Overall these data might suggest that the autonomic cardiovascular regulation is affected by acute SWCNT exposure by acting on the negative feedback mechanisms of baroreflex origin, mainly vagally mediated. These data may have relevance from an occupational perspective. In fact, given the expected widespread production of CNTs-based goods, an accidental exposure at work may be considered possible. Not surprisingly, case-reports, regarding adverse outcomes after accidental exposure to manufactured nanomaterials are starting to be reported [35-37]. Another point to be considered is that a substantial amount of workers have chronic cardiovascular disorders: these workers are highly sensitive to the adverse effects of altered cardiac autonomic regulation, and thus the effects observed in this experimental study might be seen at corresponding lower doses and might also be more severe than those reported here.

In conclusion, the results of the present study show that SWCNT pulmonary exposure might alter the physiological autonomic regulation thus contributing to cardiac and arrhythmic events as reported in response to PM and UF particles exposure.

Limitation. The generalization of the BRS response to SWCNT exposure to the whole population including also patients affected by cardiovascular diseases and in particular ischemic cardiac disease represents a limitation of this study. Therefore, it would be desirable to extend the present study to an animal model of experimentally induced myocardial infarction in order to test the hypothesis that the BRS depression induced by SWCNT exposure might also occur in patients affected by myocardial infarction.

However, considering that it has been clearly reported by clinical studies that both BRS and heart rate variability are reduced after a myocardial infarction [14] and that BRS is markedly depressed during spontaneous episodes of transient myocardial ischemia [27] and in patients without ${ }^{\sim}$ a myocardial infarction who experienced out-of-hospital ventricular fibrillation [28], it is conceivable that an eventual BRS reduction in response to SWCNT exposure in patients affected by ischemic cardiac disease might represent an environmental risk factor for developing life-threatening arrhythmic events.

Also the limited experimental sample reported in the present study might have biased some results. For this reason further experiments are needed to definitively confirm our findings.

\section{Conflict of Interests}

The authors have no conflict of interests to declare.

\section{Acknowledgments}

This study was supported in part by the Conto Terzi Grant, by Tosinvest Sanità, in part by the Ricerca Scientifica Ateneo Grant (RSA 2007-2008), and in part by and the European Commission (FP7-MARINA, Grant agreement no. 263215). 


\section{References}

[1] B. T. Mossman, P. J. Borm, V. Castranova, D. L. Costa, K. Donaldson, and S. R. Kleeberger, "Mechanisms of action of inhaled fibers, particles and nanoparticles in lung and cardiovascular diseases," Particle and Fibre Toxicology, vol. 4, p. 4, 2007.

[2] D. Liao, J. Creason, C. Shy, R. Williams, R. Watts, and R. Zweidinger, "Daily variation of particulate air pollution and poor cardiac autonomic control in the elderly," Environmental Health Perspectives, vol. 107, no. 7, pp. 521-525, 1999.

[3] C. A. Pope III, R. L. Verrier, E. G. Lovett et al., "Heart rate variability associated with particulate air pollution," American Heart Journal I, vol. 138, no. 5, pp. 890-899, 1999.

[4] D. R. Gold, A. Litonjua, J. Schwartz et al., "Ambient pollution and heart rate variability," Circulation, vol. 101, no. 11, pp. 1267-1273, 2000.

[5] S. R. Magari, R. Hauser, J. Schwartz, P. L. Williams, T. J. Smith, and D. C. Christiani, "Association of heart rate variability with occupational and environmental exposure to particulate air pollution," Circulation, vol. 104, no. 9, pp. 986-991, 2001.

[6] A. Algra, J. G. P. Tijssen, J. R. T. C. Roelandt, J. Pool, and J. Lubsen, "Heart rate variability from 24-hour electrocardiography and the 2-year risk for sudden death," Circulation, vol. 88, no. 1, pp. 180-185, 1993.

[7] Task Force of the European Society of Cardiology and the North American Society of Pacing and Electrophysiology, "Heart rate variability: standards of measurement, physiological interpretation, and clinical use," Circulation, vol. 93, pp. 1043-1065, 1996.

[8] S. S. Tsai, H. F. Chiu, T. N. Wu, and C. Y. Yang, "Air pollution and emergency room visits for cardiac arrhythmia in a subtropical city: Taipei, Taiwan," Inhalation Toxicology, vol. 21, no. 13, pp. 1113-1118, 2009.

[9] A. Peters, D. W. Dockery, J. E. Muller, and M. A. Mittleman, "Increased particulate air pollution and the triggering of myocardial infarction," Circulation, vol. 103, no. 23, pp. 28102815, 2001.

[10] K. L. Timonen, E. Vanninen, J. de Hartog et al., "Effects of ultrafine and fine particulate and gaseous air pollution on cardiac autonomic control in subjects with coronary artery disease: the ULTRA study," Journal of Exposure Science and Environmental Epidemiology, vol. 16, no. 4, pp. 332-341, 2006.

[11] Z. Li, T. Hulderman, R. Salmen et al., "Cardiovascular effects of pulmonary exposure to single-wall carbon nanotubes," Environmental Health Perspectives, vol. 115, no. 3, pp. 377-382, 2007.

[12] H. Waki, K. Katahira, J. W. Polson, S. Kasparov, D. Murphy, and J. F. R. Paton, "Automation of analysis of cardiovascular autonomic function from chronic measurements of arterial pressure in conscious rats," Experimental Physiology, vol. 91, no. 1, pp. 201-213, 2006.

[13] J. M. Legramante, S. Sacco, G. Raimondi et al., "Investigating feedforward neural regulation of circulation from analysis of spontaneous arterial pressare and herat rate fluctuations in conscious rats," American Journal of Physiology, vol. 296, no. 1, pp. H202-H210, 2009.

[14] M. T. la Rovere, G. Specchia, A. Mortara, and P. J. Schwartz, "Baroreflex sensitivity, clinical correlates, and cardiovascular mortality among patients with a first myocardial infarction: a prospective study," Circulation I, vol. 78, no. 4, pp. 816-824, 1988.

[15] M. T. la Rovere, J. T. Bigger Jr., F. I. Marcus, A. Mortara, and P. J. Schwartz, "for ATRAMI (Autonomic Tone and Reflexes After Myocardial Infarction) Investigators. Baroreflex sensitivity and heart rate variability in prediction of total cardiac mortality after myocardial infarction," The Lancet, vol. 351, no. 9101, pp. 478-484, 1998.

[16] A. A. Shvedova, E. R. Kisin, R. Mercer et al., "Unusual inflammatory and fibrogenic pulmonary responses to single-walled carbon nanotubes in mice," American Journal of Physiology, vol. 289, no. 5, pp. L698-L708, 2005.

[17] A. A. Shvedova, E. R. Kisin, R. Mercer et al., "Vitamin E deficiency enhances pulmonary inflammatory response and oxidative stress induced by single walled carbon nanotubes in C57BL/6 mice," Toxicology and Applied Pharmacology, vol. 221, no. 3, pp. 339-348, 2007.

[18] J. M. Legramante, G. Raimondi, M. Massaro, S. Cassarino, G. Peruzzi, and F. Iellamo, "Investigating feed-forward neural regulation of circulation from analysis of spontaneous arterial pressure and heart rate fluctuations," Circulation, vol. 99, no. 13, pp. 1760-1766, 1999.

[19] J. Oosting, H. A. J. Struijker-Boudier, and B. J. A. Janssen, "Validation of a continuous baroreceptor reflex sensitivity index calculated from spontaneous fluctuations of blood pressure and pulse interval in rats," Journal of Hypertension, vol. 15, no. 4, pp. 391-399, 1997.

[20] C. Mostarda, B. Rodrigues, M. Vane et al., "Autonomic impairment after myocardial infarction: role in cardiac remodelling and mortality," Clinical and Experimental Pharmacology and Physiology, vol. 37, no. 4, pp. 447-452, 2010.

[21] A. Malliani and M. Pagani, "Spectral analysis of cardiovascular variabilities in the assessment of sympathetic cardiac regulation in heart failure," Pharmacological Research, vol. 24, no. 1, pp. 43-53, 1991.

[22] E. Rondon, M. S. Brasileiro-Santos, E. D. Moreira et al., "Exercise training improves aortic depressor nerve sensitivity in rats with ischemia-induced heart failure," American Journal of Physiology, vol. 291, no. 6, pp. H2801-H2806, 2006.

[23] G. Bertineri, M. di Rienzo, A. Cavallazzi, A. U. Ferrari, A. Pedotti, and G. Mancia, "Evaluation of baroreceptor reflex by blood pressure monitoring in unanesthetized cats," American Journal of Physiology, vol. 254, no. 2, pp. H377-H383, 1988.

[24] J. M. Legramante, F. Valentini, A. Magrini et al., "Cardiac autonomic regulation after lung exposure to carbon nanotubes," Human and Experimental Toxicology, vol. 28, no. 6-7, pp. 369375, 2009.

[25] G. E. Billman, P. J. Schwartz, and H. L. Stone, "Baroreceptor reflex control of heart rate: a predictor of sudden cardiac death," Circulation, vol. 66, no. 4, pp. 874-880, 1982.

[26] P. J. Schwartz, E. Vanoli, M. Stramba-Badiale, G. M. de Ferrari, G. E. Billman, and R. D. Foreman, "Autonomic mechanisms and sudden death: new insights from analysis of baroreceptor reflexes in conscious dogs with and without a myocardial infarction," Circulation I, vol. 78, no. 4, pp. 969-979, 1988.

[27] G. Pomidossi, A. Saino, R. Perondi et al., "Impairment of the arterial baroreflex durino sympathetic and silent myocardial ischemia in humans," Journal of the American College of Cardiology, vol. 22, pp. 1866-1872, 1993.

[28] P. J. Schwartz, A. Zaza, M. Pala, E. Locati, G. Beria, and A. Zanchetti, "Baroreflex sensitivity and its evolution during the first year after myocardial infarction," Journal of the American College of Cardiology, vol. 12, no. 3, pp. 629-636, 1988.

[29] M. Piepoli, P. Sleight, S. Leuzzi et al., "Origin of respiratory sinus arrhythmia in conscious humans: an important role for arterial carotid baroreceptors," Circulation, vol. 95, no. 7, pp. 1813-1821, 1997. 
[30] J. T. Bigger Jr., M. T. la Rovere, R. C. Steinman et al., "Comparison of baroreflex sensitivity and heart period variability after myocardial infarction," Journal of the American College of Cardiology, vol. 14, no. 6, pp. 1511-1518, 1989.

[31] S. S. Hull Jr., A. R. Evans, E. Vanoli et al., "Heart rate variability before and after myocardial infarction in conscious dogs at high and low risk of sudden death," Journal of the American College of Cardiology, vol. 16, no. 4, pp. 978-985, 1990.

[32] G. M. de Ferrari, M. Landolina, M. Mantica, R. Manfredini, P. J. Schwartz, and A. Lotto, "Baroreflex sensitivity, but not heart rate variability, is reduced in patients with life-threatening ventricular arrhythmias long after myocardial infarction," American Heart Journal I, vol. 130, no. 3, pp. 473-480, 1995.

[33] S. H. Hohnloser, T. Klingenheben, A. van de Loo, E. Hablawetz, H. Just, and P. J. Schwartz, "Reflex versus tonic vagal activity as a prognostic parameter in patients with sustained ventricular tachycardia or ventricular fibrillation," Circulation, vol. 89, no. 3, pp. 1068-1073, 1994.

[34] R. E. Kleiger, J. P. Miller, J. T. Bigger Jr., A. J. Moss, and The Multicenter Post-Infarction Research Group, "Decreased heart rate variability and its association with increased mortality after acute myocardial infarction," Journal of the American College of Cardiology, vol. 59, no. 4, pp. 256-262, 1987.

[35] Y. Song, X. Li, and X. Du, "Exposure to nanoparticles is related to pleural effusion, pulmonary fibrosis and granuloma," European Respiratory Journal, vol. 34, no. 3, pp. 559-567, 2009.

[36] J. I. Phillips, F. Y. Green, J. C. A. Davies, and J. Murray, "Pulmonary and systemic toxicity following exposure to nickel nanoparticles," American Journal of Industrial Medicine, vol. 53, no. 8, pp. 763-767, 2010.

[37] T. Toyama, H. Matsuda, I. Ishida et al., "A case of toxic epidermal necrolysis-like dermatitis evolving from contact dermatitis of the hands associated with exposure to dendrimers," Contact Dermatitis, vol. 59, no. 2, pp. 122-123, 2008. 

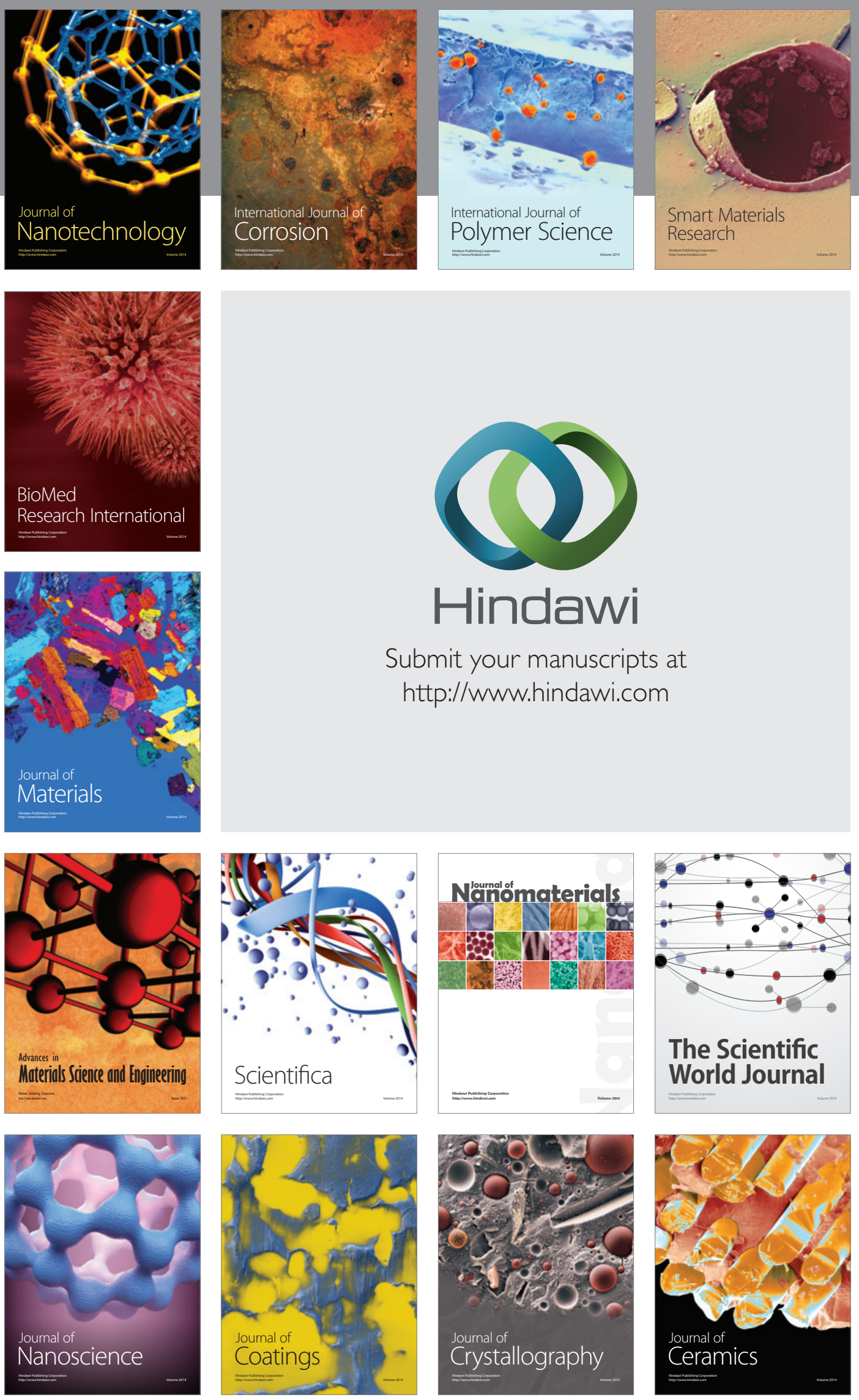

The Scientific World Journal

Submit your manuscripts at

http://www.hindawi.com

\section{World Journal}

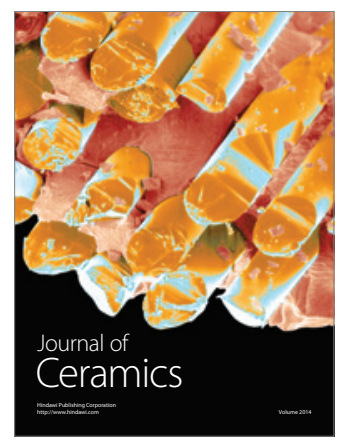

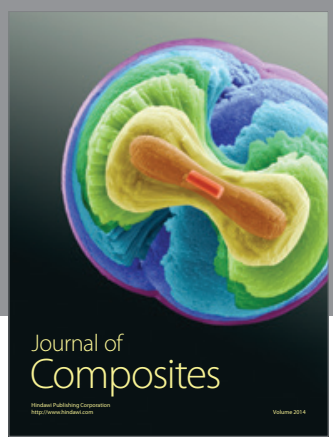
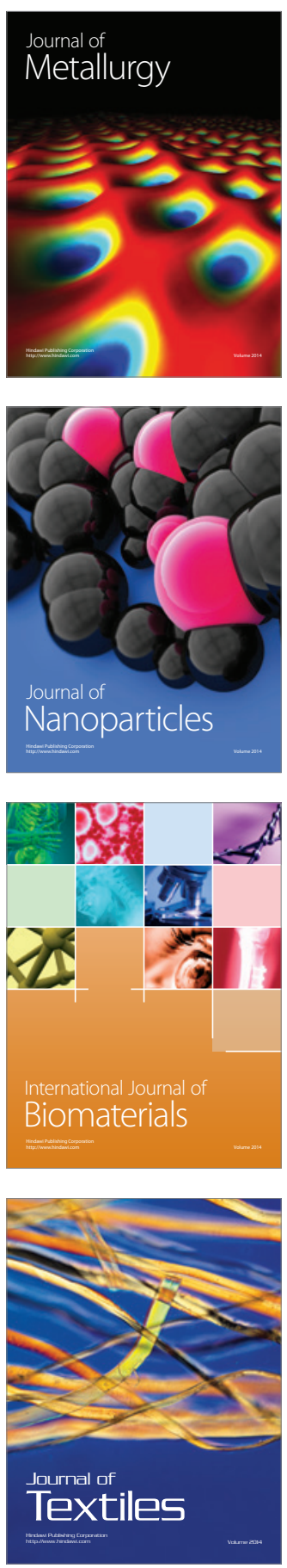